\title{
Interactive comment on "Mobile-Platform Measurement of Air Pollutant Concentrations in California: Performance Assessment, Statistical Methods for Evaluating Spatial Variations, and Spatial Representativeness" by Paul A. Solomon et al.
}

\section{Paul A. Solomon et al.}

charles.Id.blanchard@gmail.com

Received and published: 26 March 2020

Please note two corrections and one clarification to AC4. First, the overnight parking lot in Los Angeles holds $\sim 30$ vehicles. Second, there are four Google vehicles equipped with the Aclima platform. Two vehicles were available for our study, with a third available during part of the San Joaquin sampling. Of the two vehicles used throughout our study, one was equipped with methane instrumentation and one with 
a black carbon $(\mathrm{BC})$ instrument. The other two vehicles were each equipped with $\mathrm{BC}$ instrumentation, thereby providing side-by-side $\mathrm{BC}$ comparisons while parked in the San Francisco garage (Table 6). Finally, measurements of all parameters were made and recorded while vehicles were parked overnight in San Francisco and Los Angeles between $\sim 6$ - 9 a.m. before starting the drive routes and between $\sim 5-10$ p.m. after returning.

Interactive comment on Atmos. Meas. Tech. Discuss., doi:10.5194/amt-2019-501, 2020. 